\title{
Registration Technology and Morphologic Modeling of Three- dimensional Point Cloud Data
}

\author{
Xuefei Han \\ Institute of Fashion Design(Image Design), Dalian Art College, Dalian, 116021, China \\ xuefei_han@yeah.net
}

Keywords: Clothing design, Character image, Point cloud data, Model reconstruction, 3D scanning, CAD software.

\begin{abstract}
In order to improve the garment design and the character image matching effect, this paper puts forward a kind of garment character image design method based on new point cloud data, the sampling point cloud data is obtained by using the method of 3D character dense. According to the garment characters of data preprocessing and model reconstruction design, the discrete point cloud data is obtained by the 3D scanner as input and taking the object reconstruction model is as output, to construct the expression problem of 3D garment modeling obtained by measuring characters image point cloud. Finally, the 3D image of the clothing collocation characters are designed by CAD software simulation, the use of scanning point cloud character image designs two style different garment modeling, we found by contrast that second kinds of garment design is more freely with the character image, which is a very good garment sculpt design method.
\end{abstract}

\section{Introduction}

With the advent of the 3D scanning technology and the continuous development of mature, point cloud has become one of the main expression means of 3D model [1,2]. Point cloud model has the advantages of easy data acquisition and simple data structure, so it has a unique advantage to show the model with rich geometric details, such as apparel modeling and irregularly shaped objects [3]. By using the method of 3D scanning technology, this paper uses point cloud data collection to extract feature of clothing images, including initialization drawing of character, model import, model edge classification, contour line extraction and rendering as shown in Figure 1.

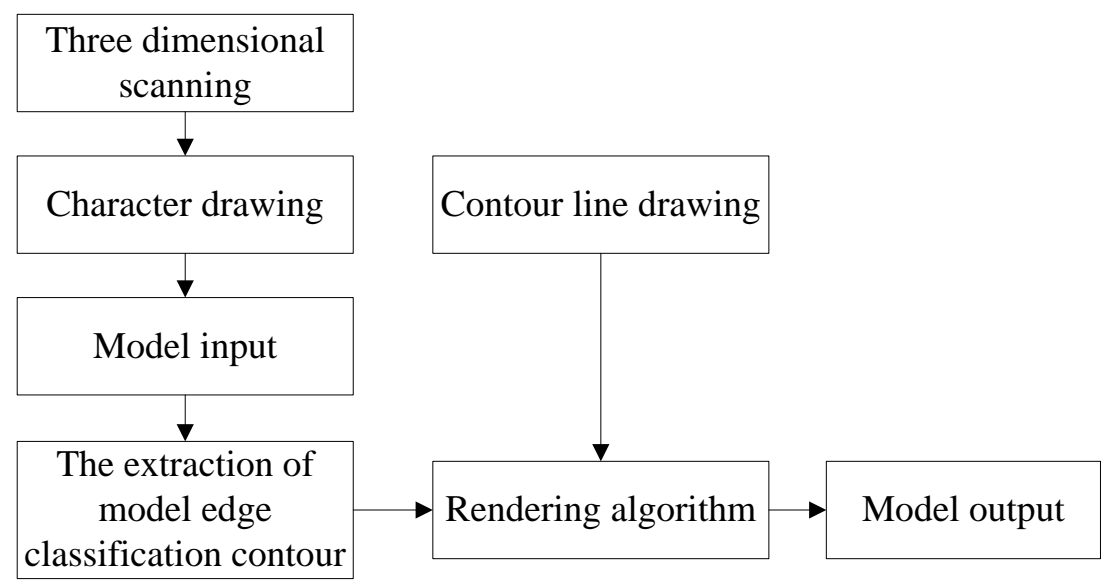

Fig.1: Schematic diagram of character image extraction implementation framework

As shown in Figure 1, in the acquisition and reconstruction of point cloud data, contour line is an important part of art style, and is a more natural way to represent the objects [4,5]. This paper uses 3D scanning technology and point cloud data acquisition method in the clothing contour line drawing and modeling design, which improves the matching ratio of garment design and the character image, which is a very good computer fashion design method. 


\section{Design of Clothing Character Contour Line Extraction Algorithm based on Point Cloud Model}

The definition of point cloud model contour points is similar to the definition of smooth surface contour points, and the contour points of point cloud model contour line is composed of point cloud model which is a collection of a series of points, these points generally meet the following conditions.

$$
n_{i} \times\left(x_{i}-C\right)=0
$$

Among them, $x_{i}$ is a point cloud model; $n_{i}$ is the normal vector of point $x_{i}$ on point cloud model; $C$ is the line of sight center, namely the position of the scanner; $\left(x_{i}-C\right)$ shows the line of sight vector, its model representation is shown in Figure 2.

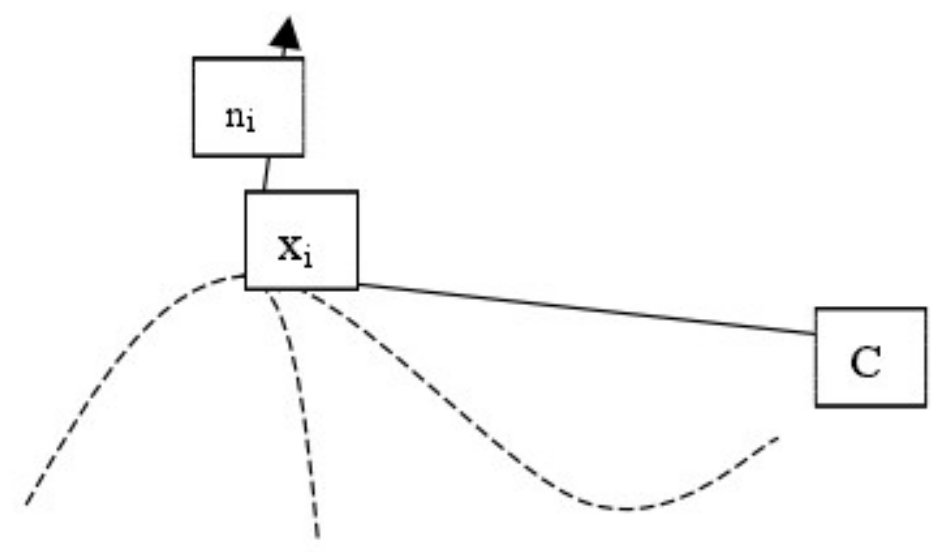

Fig.2: Schematic diagram of the contour points definition in point cloud model

As shown in Figure 2, it is the schematic diagram of the contour points definition in point cloud model, in which meeting point normal vector and sight line vector dot point product is zero, it is defined as the contour points on point cloud models, and these points can reconstruct three dimensional character image models.

$$
\begin{aligned}
& x_{i}=-i \frac{l \sin \beta}{4 \pi \beta}\left[\frac{1}{s^{3}}+i \frac{1}{s^{2}}-\frac{k^{2}}{s}\right] e^{-i k} \\
& n_{i}=-i \frac{l \cos \beta}{2 \pi \beta}\left[\frac{1}{s^{3}}+i \frac{1}{s^{2}}\right] e^{-i k}
\end{aligned}
$$

Wherein, $s$ shows the projection area, $\beta$ shows the scan angle and the expressions are as follows:

$$
k=\frac{\omega}{v}=\frac{2 \pi}{T \nu}=\frac{2 \pi}{\gamma S} .
$$

When $s \leq \leq \frac{\gamma}{6}$, there is $\frac{1}{s} \leq \frac{1}{s^{2}} \leq \frac{1}{s^{3}}$, the formula can be simplified to

$$
\begin{aligned}
& x_{i}=-i \frac{\sin \beta}{4 \pi \gamma \beta} \\
& n_{i}=-i \frac{\cos \beta}{2 \pi \gamma \beta} .
\end{aligned}
$$

When $s \geq \geq \frac{\gamma}{6}$, there is $\frac{1}{s} \geq \frac{1}{s^{2}} \geq \frac{1}{s^{3}}$, the formula can be simplified to 


$$
\begin{aligned}
& x_{i}=i \frac{1}{2 \gamma s} \sin \beta e^{-i k} \\
& n_{i}=i \frac{1}{2 \gamma s} \sin \beta e^{-i k} .
\end{aligned}
$$

In order to realize 3D garment structure characters, using the algorithm programming carries out the scanned image processing, in which part of algorithm procedure is as follows [6-8]:

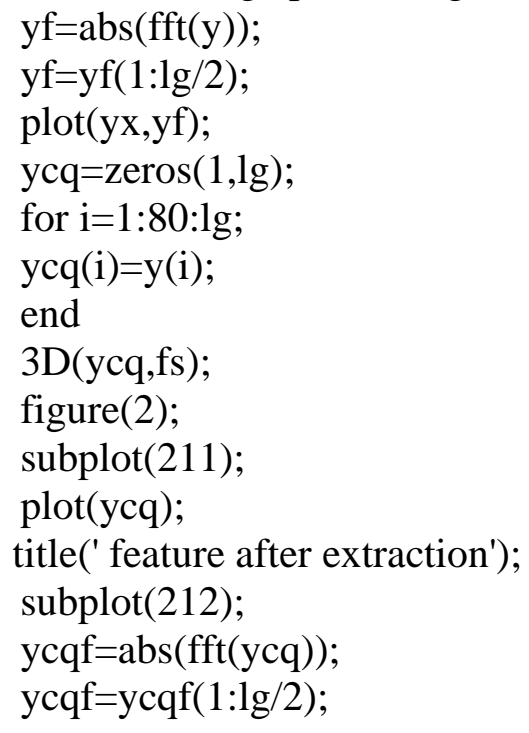

\section{The Feature Extraction of 3D Garment Character based on Point Cloud Data}

In order to verify the effectiveness and reliability of the mathematical model and algorithm for the garment character feature extraction designed in section second, this paper takes 3D garment character image design clothing as an example, the use of feature extraction algorithm extracts the 3D model of the irregular garment character figures [9-11]. The extracted schematic is shown in Figure 3.
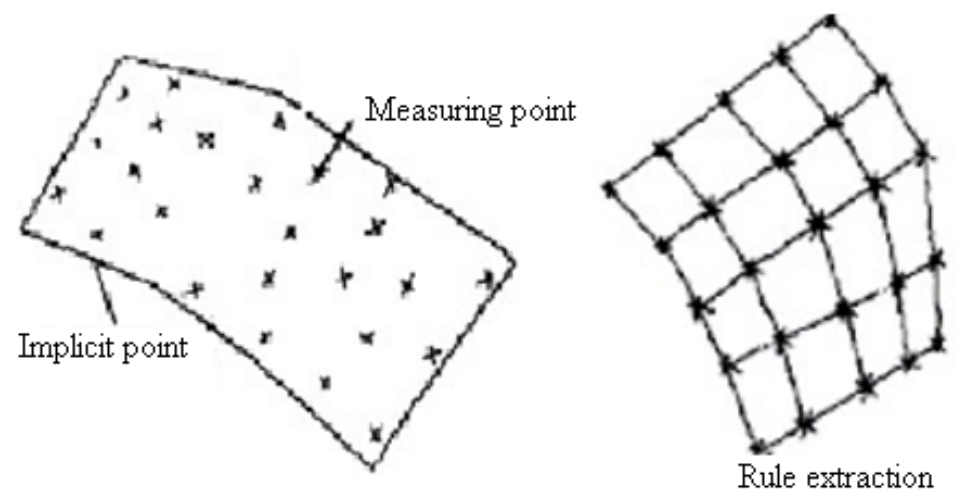

Fig.3: The sketch map 3D image extraction

With the help of simple parametric method, that is local base surface parameterization method to solve this problem [12]. The basic idea of the method is the first scattered point cloud projection to the local base surface, and then using the projection point in the local base surface parameterization instead of parametric point cloud. 


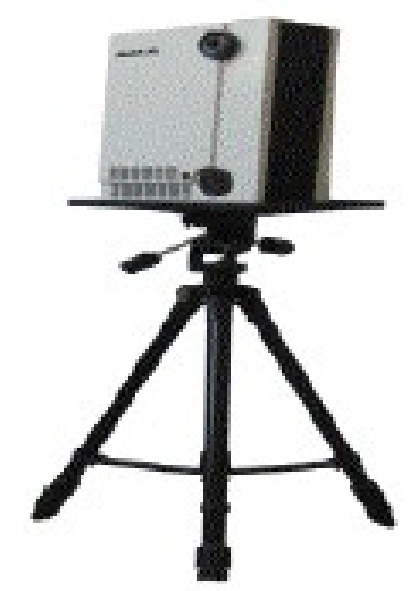

Fig.4: 3D optical scanner

As shown in Figure 4, it represents an optical scanner used in this thesis, 3D optical scanner has the advantages of fast speed, high precision, low cost, using simple, less land occupation, wide measuring range, small influenced by external environmental constraints, no damage on operator etc., its unique algorithm determines that it does not need to spray developer, it can directly measure black and dark objects, in which the output data file format is *.asc and *.dat, convenient users use CAD software to process the image.

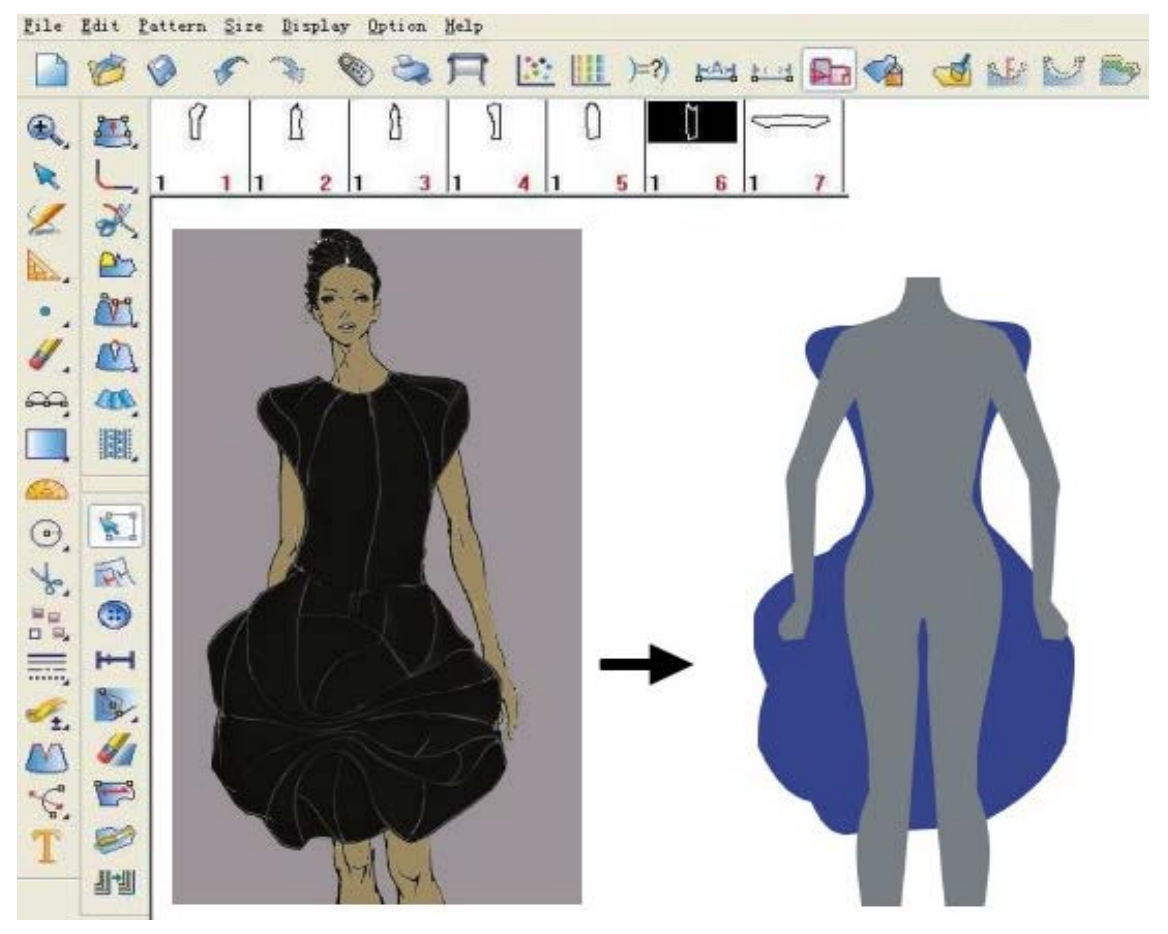

Fig.5: Clothing character image 3D design

According to the obtained character image point cloud, this paper designs two different clothing forms as shown in Figure 5. According to the character image, shoulder modeling uses internal filling means from the space construction methods, making the external profile is broad and flat, and image is more personality. 

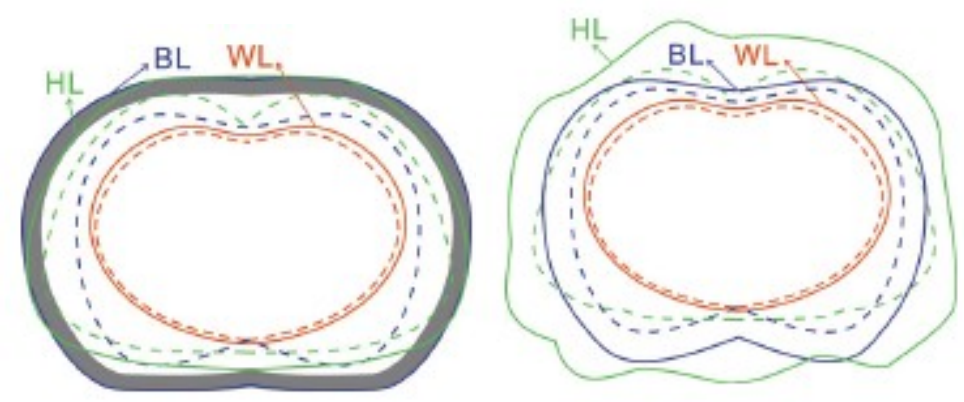

Fig.6: Level section coincidence pattern

As shown in Figure 6, it represents the horizontal section plane coincidence pattern after garment sculpt projection, it can be seen from the chart that skirt shape below waist uses many curve block surface and linear block surface, linear block surface and broken block surface etc., these does not match the formation of block surface line splicing, which shows irregular interior space form and has great random characteristics [13-15]. According to the character image, the clothing design is not only more freely, but also can design better image according to the characteristics of the time.

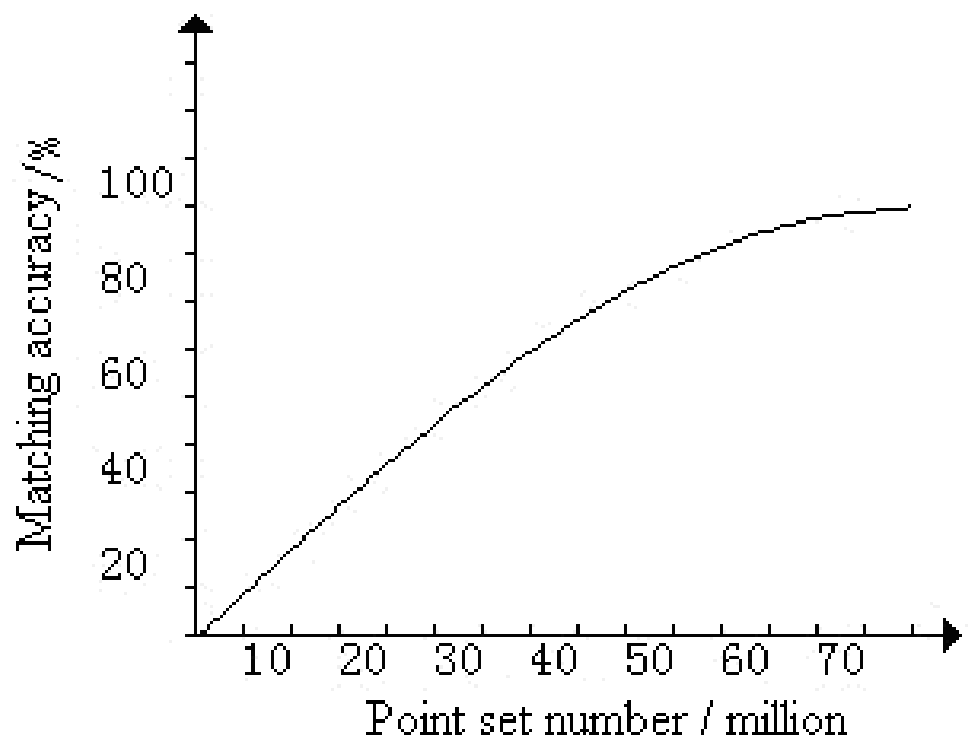

Fig.7: Characters image matching accuracy

Figure 7 shows the rate curve of garment design and character image matching, it can be seen from the chart that along with the increases of the number of 3D scanning acquisition point set, the characters image matching ratio has also increased, while the number of point set reaches more than 700000 , matching accuracy has reached more than $90 \%$, it meets the design requirements.

\section{Summary}

(1) According to the definition of point cloud model contour points and smooth surface contour points, the use of different projection area and scanning angle designs the 3D characteristics extraction mathematical model of garment character image, and the model is optimized.

(2) According to the figures point cloud obtained by scanner, this paper designs two different forms of clothing, and according to the horizontal plane section coincidence pattern on two sets of clothing to carry on evaluation, we can get the characters and clothing matching rate curve, which provides a new computer method for clothing design.

\section{References}

[1] R. Wang, J.S. Li, L.X. Liu, R. Luo. The point cloud registration algorithm based on geometric feature.Journal of East China University of Science and Technology, 2012(4): 24-28. 
[2] L.F. Kong, D.Z. Yang, S.H. Zhang. The point cloud automatic registration of 3D vision reconstruction system based on parallel mechanism. Journal of Yanshan University, 2011(2): 78-81.

[3] K.B. Yang, X. Li, X.Y. Zhang, C.T. Wang. Study of the scattered point cloud data Kneighborhood fast search algorithm. Agricultural equipment and vehicle engineering, 2011(3): 34-38.

[4] X.C. Zhang, J.T. Xi, J.Q. Yan. Point cloud data and CAD model registration based on the extended Gauss ball. Journal of mechanical engineering, 2011(2): 81-84.

[5] J.J. Wang Jingju, J. Wang Jian. The elements analysis that influence the spatial variation. Garment decoration, 2011(1): 112-113.

[6] L.L. Ma. Clothing space design. Progress of textile technology, 2013(1): 91-95.

[7] Y. Zhang. The beauty characteristics of fashion show. The beauty and age, 2013(4): 41-42.

[8] X.C. Wang, J.J. Cao, X.P. Liu, B.J. Li. Application of the wave front method in repairing holes in triangular meshes. Journal of computer aided design and graphics, 2011(2): 49-50.

[9] X.L. Wei, B.X. Xiao, X.Y. Guo, W.L. Wen, S.L. Lu. Application of 3D laser scanning technology in plant scanning analysis. China Agricultural Science Bulletin, 2012(4): 45-48.

[10] C.J. Zhao, S.L. Lu, X.Y. Guo, B.X. Xiao, W.L. Wen. The discussion of digital plant and its technology system. Chinese agricultural science, 2012(6): 34-37.

[11] G.P. Qian, R.F. Pan, R.F. Tong. Mesh repairs of keep the features. Chinese image journal graphics, 2011(6): 23-26.

[12] X.D, H.G. Cui, H.Y. Hu. The point cloud simplification algorithm based on curvature feature. Computer applications, 2011(2): 98-99.

[13] S.L. Lu, X.Y. Guo, C.F. Li. A precise modeling and rendering of 3D plant leaves. China Journal of image and graphics, 2012(4): 136-137.

[14] L. Yang, X.Y. Guo, S.L. Lu, C.J. Zhao. Cucumber leaf shape 3D reconstruction based on multiple images. Journal of agricultural engineering, 2011(2): 78-80.

[15] X.N. Li, W.Y. Wu, M. Wei. Application of geometric features in the point cloud registration. Computer engineering and application, 2012, 2(32): 45-46. 